\title{
Patients face five hour delays after private firm takes over transport service
}

To illustrate a shortened version of this story ( $B M J$ 2016;353:i2153, doi:10.1136/bmj.i2153) in the "Seven Days in Medicine" section in the print issue of The BMJ of 23 April, we used a photograph of an ambulance from the Special Ambulance Transfer Service Ltd. The photograph was inappropriate in two ways:
- The service referred to in the story was run by Coperforma and not by the Special Ambulance Transfer Service Ltd.

- The ambulance pictured has been out of service since 2007.

We would like to apologise for the inappropriate use of this image and any confusion it may have caused. 